EVS26

Los Angeles, California, May 6-9, 2012

\title{
Low-cost FC Stack Concept with Simplified Configuration Utilizing Advanced MEA and FC Module
}

\author{
Mitsutaka Abe, Keiji Ichihara, Takanori Oku, Kazuhiro Kageyama, Yasuhiro Numao and \\ Masanari Yanagisawa
}

EV System Laboratory, Nissan Research Center, Nissan Motor Co., Ltd. 1, Natsushima-cho, Yokosuka-shi, Kanagawa 237-8523, Japan (E-mail: mitsu-abe@mail.nissan.co.jp)

\begin{abstract}
The development of fuel cell vehicles (FCVs) at Nissan proceeded to the level of limited customer leasing with the X-TRAIL FCV equipped with an in-house FC stack (2005- model). Subsequently, a further improved 2008-model FC stack was developed with double the power density and using only half the amount of precious-metal catalysts, thus giving the vehicle subzero startability at $-20^{\circ} \mathrm{C}$. For the coming stage of FCV commercialization, we have proposed the concept of a compact and low-cost FC stack (2011 model) that increases the maximum power density to $2.5 \mathrm{~kW} / \mathrm{L}$ and reduces the estimated production cost to a level that comes very close to meeting the target of the DOE Hydrogen Program for the adoption of 2010 technologies. These advances were achieved mainly by improving membrane electrode assembly (MEA) performance and simplifying the FC stack structure. This paper presents an overview of the simplified structure of the new FC stack incorporating various new features: a shell-enclosed FC module and simplified configuration. This remarkable evolution of the FC stack structure was brought about by the development of various technologies: an advanced MEA, an adhesive seal, and a fastening enclosure. The advanced MEA has an integrated molded frame around it to reduce the production cost. Another benefit of the advanced MEA is the ability to build a stiff insulating shell around a FC module when the frames are bonded together with adhesive seals to form the module. As a result, the FC module can be fastened directly using steel plates uniquely shaped in both the stacking direction and the direction perpendicular to it, thereby maintaining proper compression to prevent the stacked FC modules from sliding. These improvements reduce the variety of components of the new FC stack by an estimated 75\%, thus increasing the maximum power density by 30\%, compared with the 2008-model FC stack.
\end{abstract}

Keywords: fuel cell, hydrogen, PEM fuel cell (proton exchange membrane) 


\section{Introduction}

\subsection{FCV Development Plan in Nissan Green Program}

The Nissan Green Program was implemented as a means of achieving the company's environmental philosophy of symbiosis of people, vehicles and nature. A new six-year environmental action plan, Nissan Green Program (NGP 2016), was announced in October 2011 [1] that focuses on reducing the environmental impact of corporate activities and pursuing harmony between resource consumption and ecology by promoting and widening the application of green technologies that were developed in NGP 2010, the previous environmental action plan, and contributing to a recycling-based society. In line with the aim of the company's medium-term environmental plan to be No. 1 in zero-emission vehicles, it is planned to develop and introduce an all-new fuel cell vehicle (FCV).

\subsection{History of FCV Development at Nissan}

The development of FCVs at Nissan has proceeded to the level of limited customer leasing as shown in Figs. 1 and 2. Limited leasing and intensive field tests of FCVs were started using the X-TRAIL FCV equipped with an in-house FC stack (2005 model) [2]. By analyzing the data obtained in real-world driving tests, the major causes and mechanisms of performance decay were determined and that valuable information was used to accelerate the development of FC operating modes and the materials of the membrane electrode assembly (MEA) [3, 4].

The FC stack was improved further with the 2008-model stack that doubled power density and halved the amount of precious-metal catalysts used [5], thus giving the X-TRAIL FCV subzero startability at $-20^{\circ} \mathrm{C}[6,7]$. In addition, the impact on performance decay under a condition of higher current density was investigated, with the aim of further downsizing the FC stack and reducing catalyst loading. By utilizing an equivalent circuit model, the phenomena involved and dominant factor of catalyst degradation were identified [8].

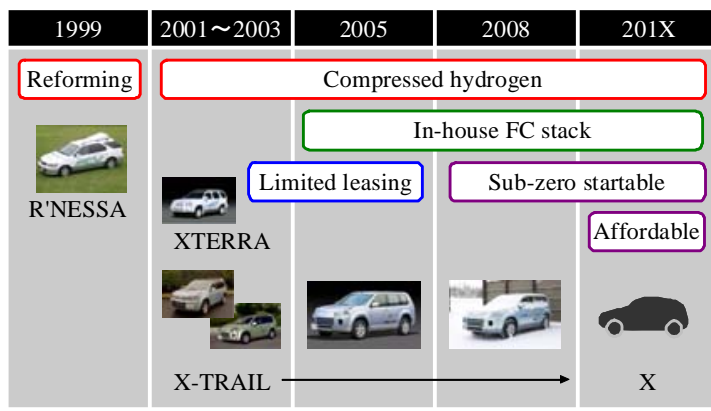

Figure 1: History of Nissan FCV Development on Model-year Basis

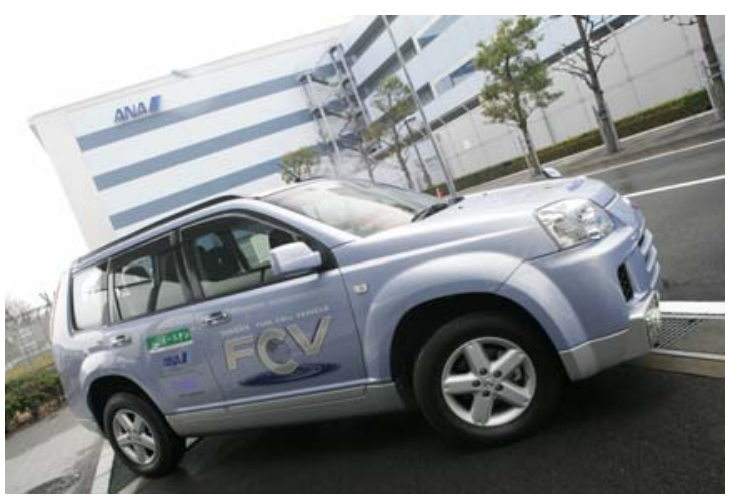

Figure 2: Demonstration of X-TRAIL FCV as Airline Courtesy Car Operated by HySUT [9]

\subsection{Newly Developed FC Stack}

For the coming stage of FCV commercialization, we have proposed a new FC stack concept (2011 model) as shown in Fig. 3, which incorporates various new technologies such as an advanced MEA [10-12]. With increased maximum power density of $2.5 \mathrm{~kW} / \mathrm{L}$ as shown in Fig. 4, this new FC stack is expected to be compact enough to be applied to a wide range of vehicles. In addition, the mass production cost of the stack is expected to be significantly reduced to a level that comes very close to meeting the target of the U.S. DOE Hydrogen Program for the adoption of 2010 technologies $[13,14]$ as shown in Fig. 5.

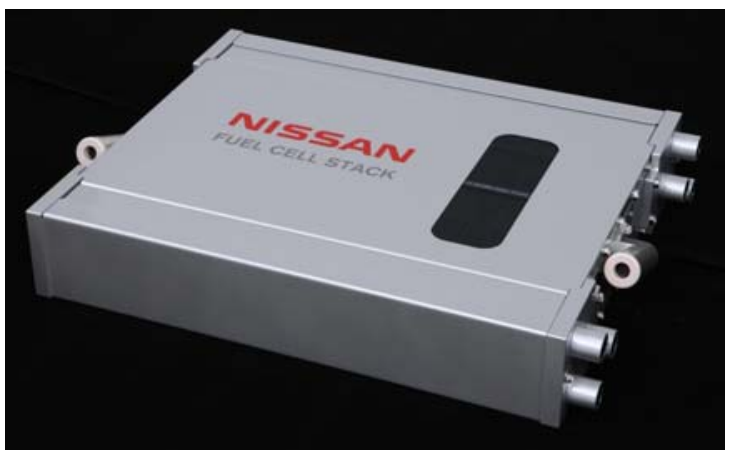

Figure 3: 2011-model Nissan FC Stack 


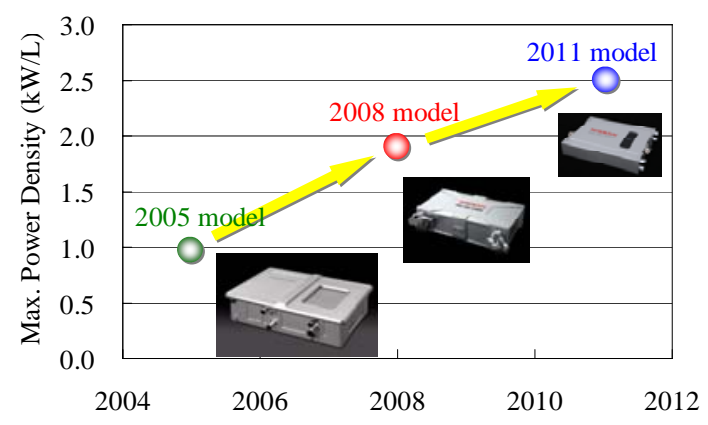

Figure 4: Evolution of Nissan FC Stack in Maximum Power Density

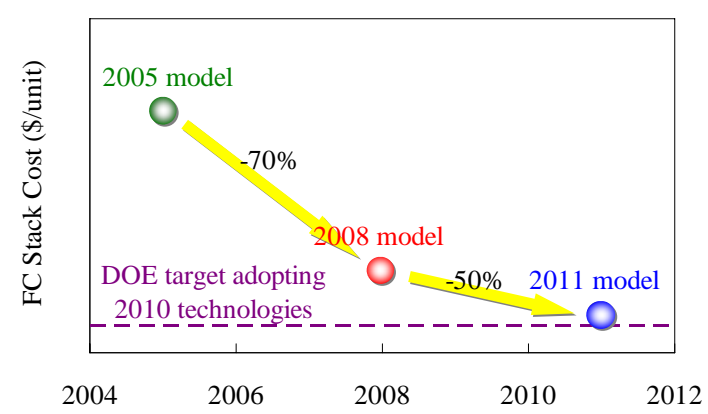

Figure 5: Evolution of Nissan FC Stack in Mass Production Cost: Compared with the DOE target adjusted to be consistent with the production volume and components included.

This progress toward a compact and low-cost FC stack was achieved mainly by improving MEA performance and simplifying the FC stack structure. Since the improved MEA performance in terms of higher maximum current density has been described elsewhere [8, 10 and 11], this paper focuses on the simplified stack structure and describes various new technologies developed.

\section{Overview of Simplified Structure of New FC Stack}

\section{$2.1 \quad$ Features of Simplified Structure}

The configuration of the new FC stack structure is shown in Fig. 6. A conventional FC stack is composed of hundreds of cells assembled with end plates and fastening plates in a multi-row configuration. In addition, the FC stack is generally placed in a costly vessel-type enclosure. The new FC stack has been developed to simplify this complicated structure and associated production process. The features of the new FC stack structure are twofold:

(1) Shell-enclosed FC module
(2) Simplified single-row configuration and a plate enclosure

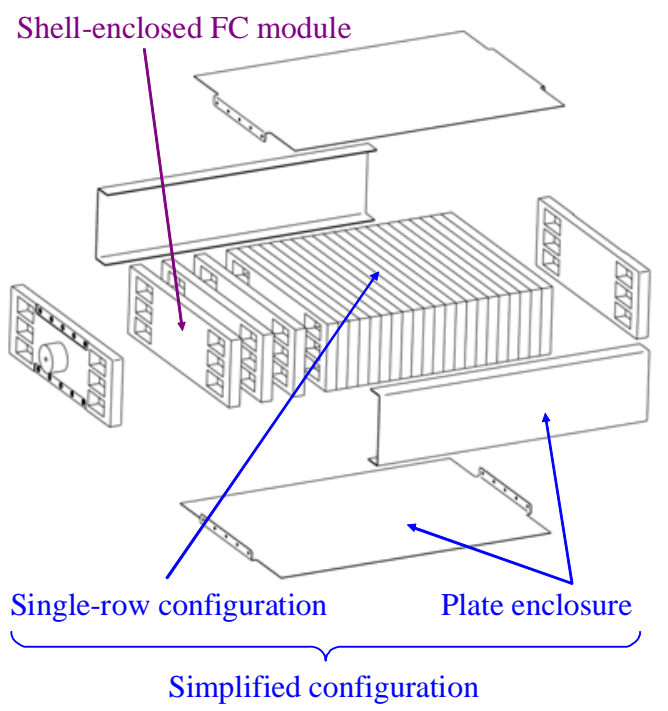

Figure 6: Configuration of Simplified FC Stack

In the process of making the new FC stack, a dozen cells are first assembled to form a FC module that is enclosed in a stiff insulating shell. One of the benefits of using the FC module is that it allows easy stacking and replacement of hundreds of cells. Individual FC modules are then stacked in a single row, allowing the elimination of such components as manifolds and bus bars normally required for a multi-row configuration. Furthermore, instead of using a conventional vessel-type enclosure, the fastening plates around the FC stack also serve as an enclosure. As a result, the variety of components of the new FC stack is reduced by $75 \%$ compared with the 2008 -model FC stack as shown in Fig. 7.

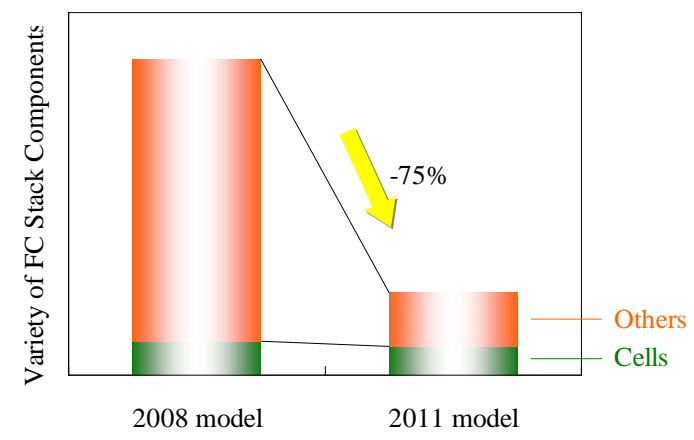

Figure 7: Effect of Simplified Structure on Reducing the Variety of FC Stack Components 


\subsection{Technologies Developed for Simplified Structure}

This remarkable evolution of the FC stack structure has been driven by the development of following technologies:

(1) Advanced MEA and adhesive seals for the shell-enclosed FC module

(2) Fastening enclosure for the simplified configuration

The shell-enclosed FC module is achieved with the integrally molded frame (IMF) of the MEA and adhesive seals. The MEA has a plastic frame molded around it, reducing the production cost. Another benefit of the advanced MEA is the ability to build a stiff insulating shell around a FC module by bonding the frames together with adhesive seals to form a module. This allows a FC module to be fastened directly using steel plates uniquely shaped in both the stacking direction and the direction perpendicular to it, thereby maintaining proper compression to prevent the stacked FC modules from sliding. Furthermore, since the shells around FC modules act as an inner enclosure, the combination of fastening plates is sufficient to form an outer fastening enclosure.

\section{Shell-enclosed FC Module}

\subsection{MEA with IMF}

Two technologies were adopted for fabricating FC modules: a MEA with IMF and adhesive seals. A conventional MEA, including that of the 2008-model FC stack has been supported by a frame composed of laminated plastic sheets on both sides of the MEA rim. In this study, an IMF was developed as shown in Fig. 8, not only to simplify the MEA structure and production process for cost reductions, but also to form a raised projection around the IMF rim that can be utilised for making a FC module. The IMF is formed by plastic injection molding and simultaneously joined to a MEA by means of a joint that is formed by impregnating the MEA rim with molten plastic.

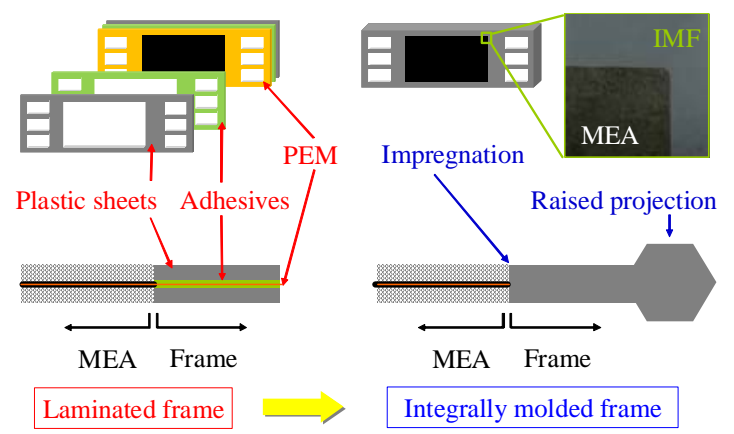

Figure 8: Newly Developed MEA with IMF

One of the technical challenges of the IMF is control of IMF impregnation; over-impregnation results in reducing not only the MEA reaction area but also joint strength uniformity. The desired structure and properties have been obtained by optimizing the MEA characteristics, plastic properties and molding conditions. As a result, tests have confirmed that the adoption of this IMF has little influence on either MEA performance or durability.

\subsection{Adhesive Seals}

The other key technology adopted for fabricating FC modules is the adhesive seals that enable cells to be sealed and assembled as shown in Fig. 9. For a conventional FC stack, including the 2008-model FC stack, the gap between a MEA and separators was sealed using compressive seals such as gaskets. Since stacked individual cells were unstable and easily slid before compression was applied or after it was removed, careful handling was required for both assembly and replacement of hundreds of cells.

In this study, adhesive seals were adopted for sealing the gap between a MEA and separators. In addition, the raised projection of the IMF are also bonded to each other with the same adhesive, which allows a dozen individual cells to be included in a single FC module. Furthermore, the bonded IMFs form a shell around the FC module because the materials of both the IMF and adhesive seals have sufficient isolation properties for preventing the leakage of electricity, hydrogen and water. As a result, the shells achieve a simple configuration, enabling hundreds of single cells to be handled easily and stacked stably. 


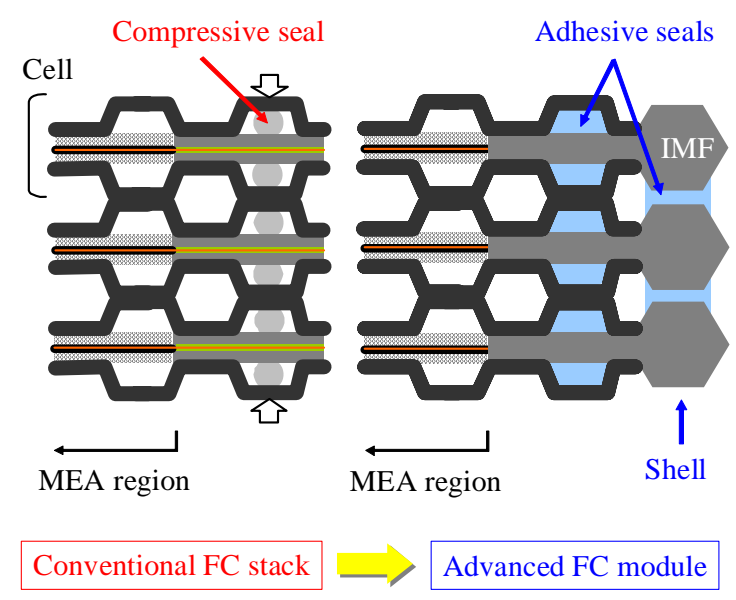

Figure 9: Shell-enclosed FC Module Formed by IMFs and Adhesive Seals

\section{Simplified Configuration}

\subsection{Fastening Enclosure}

Previous FC stacks, including the 2008-model FC stack, have been surrounded by a costly vessel-type enclosure to prevent leakage of electricity, hydrogen and water. With the adoption of the advanced FC module, the bonded IMFs act as a shell around the module as shown in Fig. 9, not only for insulation, but also for maintaining sufficient module stiffness. Thanks to these properties, FC modules can be fastened directly using steel plates in both the stacking direction and the direction perpendicular to it, thereby stabilizing modules so that they do not slide even under vibrating conditions of vehicle operation.

To take advantage of these FC module properties, steel plates with a unique shape were developed to serve as fastening plates as well as to form the outer fastening enclosure. This fastening enclosure is composed of tension plates and holding plates as shown in Fig. 10. By adopting both the fastening enclosure and shell-enclosed FC modules, more than 400 individual cells can be stacked in a single row without sliding, while eliminating such components as manifolds and bus bars usually required for a multi-row configuration. In addition, the plates can also be utilized as an alternative enclosure as shown in Fig. 6 and Fig. 10 because complete sealing for this outer enclosure is not required thanks to the FC module shell as an inner enclosure. Therefore, the costly box-type enclosure required for a conventional FC stack can also be eliminated.

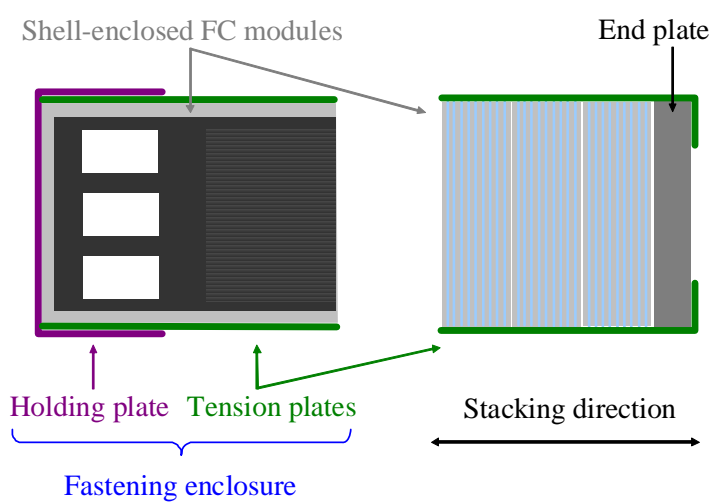

Figure 10: Fastening Enclosure for Both Single-row Configuration and Alternative Enclosure

\subsection{Effect of Simplified Structure of New FC Stack}

As a result of this simplification of the FC stack structure, the variety of components is reduced by 75\% compared with the 2008-model FC stack (Fig. 7). Thanks to both the simplified structure achieved in this study and increased current density [8, 10 and 11], it is estimated that the maximum power density of the new FC stack will be increased by $30 \%$ over the previous stack, reaching $2.5 \mathrm{~kW} / \mathrm{L}$ as shown in Fig.3. The compactness of the FC stack achieved in this study indicates greater flexibility with respect to the vehicle size and onboard location such as the battery bay of EVs or the engine compartment of internal combustion engine vehicles.

\section{Conclusions}

For the coming stage of FCV commercialization, the concept of a compact and low-cost FC stack was proposed, which incorporates various new features such as a shell-enclosed FC module, a simplified single-row configuration, and a plate enclosure. With the simplified structure and increased current density, the variety of components is reduced by $75 \%$ and the maximum power density is increased to $2.5 \mathrm{KW} / \mathrm{L}, 30 \%$ higher than the 2008-model FC stack. Although this new FC stack is expected to be compact enough to be applied to a wide range of vehicles, further cost reduction efforts such as through the attainment of higher current density and lower catalyst loading are necessary to make FCVs more affordable to ordinary consumers. The progress described here illustrates some of the critical FC stack developments attained at Nissan and shows 
the dedication and determination to be No. 1 in zero-emission vehicles.

The technologies developed in this study for simplifying the FC stack structure are summarized below:

(1) A MEA with an IMF produced by injection molding has been adopted instead of a conventional frame composed of laminated sheets. The IMF contributes not only to simplifying the MEA structure and production process for cost reductions, but also to forming a raised projection for use in fabricating FC modules. Tests results have confirmed that the adoption of this IMF has little influence on either MEA performance or durability.

(2) An adhesive seal has been adopted instead of a conventional compressive seal for bonding a dozen individual cells to form a single module structure. A FC module is enclosed in a stiff insulating shell built by bonded IMFs. The shells achieve a simple configuration, enabling hundreds of single cells to be handled easily and stacked stably

(3) A fastening enclosure built with fastening plates has been adopted. It keeps stacked FC modules stable and prevents them from sliding, thereby enabling a single-row configuration of more than 400 individual cells. In addition, it also acts as an alternative outer enclosure that does not require complete sealing, thanks to the insulation properties of FC module shells. This eliminates the need for a costly vessel-type enclosure used for conventional FC stacks.

\section{Acknowledgments}

The MEA technologies described here were developed jointly with W. L. Gore \& Associates, Co., Ltd. The authors wish to thank everyone involved at W. L. Gore for their valuable assistance.

\section{References}

[1] Nissan Green Program 2016, http://www.nissanglobal.com/EN/ENVIRONMENT/APPRO ACH/GREENPROGRAM/, October 24, 2011

[2] T. Aoyama, A. Iiyama, K. Shinohara, S. Kamegaya, S. Yamamoto, and Y. Ban,
Status of FCV Development at Nissan and Future Work, SAE, 2008-01-0423, 2008

[3] R. Shimoi, T. Aoyama, and A. Iiyama, Development of Fuel Cell Stack Durability Based on Actual Vehicle Test Data: Current Status and Future Work, SAE, 2009-01-1014, 2009

[4] R. Shimoi, T. Aoyama, A. Matsunaga, and A. Iiyama, Development of a Method for Estimating the Durability of Fuel Cell Stacks for Vehicle Use, JSAE Annual Congress (Spring), Proceedings No. 22-09, 20095032, 2009

[5] A. Miyazawa, K. Ikezoe, Y. Okuyama, and M. Yanagisawa, The Development of High Power Density and Low Cost New Fuel Cell Stack, JSAE Annual Congress (Spring), Proceedings No. 22-09, 109-20095161, 2009

[6] K. Ikezoe, Y. Tabuchi, F. Kagami, and H. Nishimura, Development of a FCV with a New FC Stack for Improved Cold Start Capability, SAE, 2010-01-1093, 2010

[7] K. Ikezoe, Y. Tabuchi, F. Kagami, and H. Nishimura, Development of a New FC Stack with Improved Cold Start Capability, JSAE Annual Congress (Spring), Proceedings No. 25-10, 20105172, 2010

[8] S. Takaichi, M. Abe and Y. Yanagisawa, Development of Low-cost FC Stack Drability with High Power Density, JSAE Annual Congress (Spring), Proceedings No. 88-11, 20115129, 2011

[9] HySUT, the Research Association of Hydrogen Supply/Utilization Technology, http://hysut.or.jp/en/index.html, July 31, 2009

[10] M. Abe, T. Oku, Y. Numao, S. Takaichi and Y. Yanagisawa, Low-Cost FC Stack Concept with Increased Power Density and Simplified Configuration Utilizing an Advanced MEA, SAE International Journal of Engines, June 20114 (1), 1872-1878

[11] M. Abe, T. Oku, Y. Numao, S. Takaichi and Y. Yanagisawa, Low-cost FC Stack Concept with Increased Power Density and Simplified Configuration -Utilizing an Advanced MEA with Integrated Molded Frame-, EVTeC'11, 20117260, 2011

[12] Nissan Develops Next Generation Fuel Cell Stack, $\quad$ http://www.nissanglobal.com/EN/NEWS/2011/_STORY/11101 3-01-e.html, October 13, 2011

[13] Hydrogen, Fuel Cells and Infrastructure Technologies Program - Multi-year Research, Development and Demonstration Plan - 3.4 Fuel Cells 2007, 
http://www1.eere.energy.gov/hydrogenandf uelcells/mypp/pdfs/fuel_cells.pdf, April 2009

[14] B. James, J. Kalinoski, and K. Baum, MassProduction Cost Estimation for Automotive Fuel Cell Systems, DOE Hydrogen Program Review, Project ID \#FC018, 2010

\section{Authors}

\section{Mr. Mitsutaka ABE}

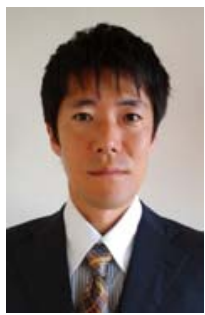

EV System Laboratory, Nissan

Research Center, Nissan Motor Co.,

Ltd.

1, Natsushima-cho, Yokosuka-shi

Kanagawa 237-8523, Japan

Tel: +81-46-867-5378

Fax: +81-46-867-5332

Email: mitsu-abe@mail.nissan.co.jp 